\title{
Determination of $\alpha$-Resolution for Lattice-Valued First-Order Logic Based on Lattice Implication Algebra
}

\author{
Yang $\mathrm{Xu}^{1}$ Xiaobing $\mathrm{Li}^{1} \mathrm{Jun} \mathrm{Liu}^{2}$ Da Ruan ${ }^{3}$ \\ ${ }^{1}$ Department of Mathematics, Southwest Jiaotong University, Chengdu 610031, P.R. China \\ ${ }^{2}$ School of Computing and Mathematics, Faculty of Engineering, University of Ulster at Jordanstown, \\ Newtownabbey BT37 0QB, Northern Ireland, UK \\ ${ }^{3}$ The Belgian Nuclear Research Centre (SCK•CEN), Boeretang 200, B-2400 Mol, Belgium
}

\begin{abstract}
As a continuation of our research work on resolutionbased automated reasoning approaches for latticevalued logic systems with truth-values in a latticevalued logical algebraic structure - lattice implication algebra (LIA), in the present paper, we prove that $\alpha$ resolution for lattice-valued first-order $\operatorname{logic} L F(X)$ based on LIA can be equivalently transformed into that for lattice-valued propositional logic $L P(X)$ based on LIA, and then prove that $\alpha$-resolution for latticevalued propositional $\operatorname{logic} L_{n 2} P(X)$ which is linguistic truth-valued propositional logic based on a lattice implication algebra based on LIA can be equivalently transformed into $\alpha$-resolution for lattice-valued propositional logic $L_{n} P(X)$ based on another linguistic-valued LIA. Finally, the determination table of $\alpha$-resolution of any two generalized literals under 49 cases for lattice-valued propositional $\operatorname{logic} L P(X)$ is given so that the determination of $\alpha$-resolution for lattice-valued first-order logic $L F(X)$ as a key issue, can be accordingly resolved, which, at the same time, provide the key support for $\alpha$-resolution automated reasoning algorithms in linguistic truth-valued logic based on LIA.
\end{abstract}

Keywords: Lattice implication algebra, Lattice-valued propositional logic $L P(X)$, Lattice-valued first-order logic $L F(X)$, Linguistic truth value, $\alpha$-resolution

\section{Introduction}

It is well known that incomparability is a kind of uncertainty often associated with human's intelligent activities in the real world, and it exists not only in the processed object itself, but also in the course of the object being dealt with. In fact, it is a kind of overall uncertainty of objects caused due to the complexity of objects itself, associated with many factors and the inconsistent token among those factors each other presents overall uncertainty of objects. Hence, the incomparablity occurs inevitably during dealing with the complex objects.

In order to deal with incomparability, we started investigation from 1993 from the symbolism point of view based on the following academic routine: based on symbolism from 1993: logical algebra - lattice implication algebra (LIA)[1], algebric logic - latticevalued logic based on LIA [3, 4, 12-14], approximate reasoning - uncertainty reasoning and automated reasoning in lattice-valued logic based on LIA $[5,10$, 15]. One of the fundamental goals is to provide scientific and reasonable logical framework and practical and efficient inference methods based on appropriate symbolic logical system for dealing with both comparability and incomparability in the intelligent information processing.

This paper aims at resolution-based automated reasoning under incomparability in lattice-valued firstorder logic $L F(X)$ based on LIA. Since the introduction of resolution principle in 1965 by Robison [19], automated reasoning based on resolution principle for classical logic has been extensively studied[19-21, 30-33], which has the following three obvious characteristics: firstly, forms of literals are simple, and literals usually contain no constants and implication connectives; secondly, there is only a kind of resolution, that is $O$-resolution; thirdly, it is easily to judge if two literals is $O$ resolvent, that is to judge if the two literals are complementary pair. There have also been many investigations on resolution-based automated reasoning in many-valued logical framework $[16,22$, 34]. In essence, this kind of resolutions is to transform the resolution in many-valued logic to the resolutionbased automated reasoning in classical logic. How to deal with incomparability, which is complex and not described by chain-type logic, leads to the complexity of logical formula in lattice-valued logic based on lattice implication algebra. Correspondingly the logical formula have the following new characteristics: (1) there exist generalized literals which contain constants and implication connectives; (2) resolution 
level $\alpha$ can be chosen from truth-valued fieldlattice implication algebra $L$, that is $\alpha$-resolution; (3) it is difficult to judge if two generalized literals are resolvent. Because of these new characteristics, it is not feasible to directly apply the resolution-based automated reasoning theory and methods for classical logic and chain-type many-valued logic into that of lattice-valued logic with incomparability. Hence, since 1996, we started investigation on resolution-based automated reasoning with incomparability in latticevalued logic based on lattice implication algebra. $\alpha$ resolution principle based on lattice-valued propositional logic $L P(X)$ are established [5,7]. Later, the $\alpha$-resolution algorithms are discussed based on lattice-valued propositional logic $L P(X)[6,8,17,18$, 23]. Resolution-based automated reasoning methods based on filters for lattice-valued propositional $\operatorname{logic} L P(X)$ are proposed [24, 26].

On the other hand, the course of human behavior or thinking resolving some real problems can be treated as a proof of soft theorems(some conclusions with uncertainty), which a lot of natural language reasoning got involved. Therefore, it is necessary to study theories and approaches of linguistic-valued based automated reasoning under uncertainties during the investigation of the machine intelligence. In [25], some opinions on fuzzy logic from the view of machine intelligence are presented, characteristics of fuzzy logic that adapted to investigate machine intelligence are analyzed, and the following opinions are proposed: (1) machine intelligence requires uncertain reasoning with linguistic expressions; (2) study uncertain reasoning based on logic is one of scientific methodologies; (3) machine intelligence demands uncertain reasoning with linguistic information under the guidance of logic; (4) machine intelligence also requires automated reasoning with linguistic information based on logic. In order to establishing algebraic structure of linguistic truth values, linguistic truth-valued lattice implication algebra [9] is established and its properties are discussed. In [11], weak completeness of $\alpha$ resolution in a linguistic truth-valued propositional logic based on lattice implication algebra are discussed. In [27], a linguistic truth-valued propositional logic based on LIA is proposed, the properties of logical formula in it are given, $J$-true, $J$-false, $J$-similar and $J$-complementary literals based on filters are defined and the $J$-resolution method are given. A soft resolution method in linguistic truth-valued first-order logical system with six elements based on lattice implication algebra is proposed [29].

In order to establish practical and efficient resolution-based automated reasoning methods under incomparability based on lattice-valued logic, the key problem is to judge the $\alpha$-resolution of any two generalized literals, i.e., their resolution at certain resolution level.

In this paper, we finally provide with a determination table from which we can clearly and directly get the resolvability among generalized literals at some resolution level. Hence, the key problem for the determination of $\alpha$-resolution of lattice-valued first-order logic $L F(X)$ based on lattice implication algebra is basically resolved, which provide key support as well for establishing resolution-based automated reasoning algorithms of linguistic truth-valued logic.

The remainder of the paper is organized as follows: in Section 2, the proofs that $\alpha$-resolution in $L F(X)$ can be equivalently transformed into that in $L P(X)$ and $\alpha$-resolution in $L_{n 2} P(X)$ can be equivalently transformed to that in $L_{n} P(X)$ are given. In Section 3, four kinds of typical inequality of generalized literals are given. In Section 4, $\alpha$ resolution determination table under 49 cases between two generalized literal is given. The paper is concluded in Section 5.

\section{Transformation of $\alpha$-resolution}

Definition 2.1[11] Let $\alpha \in L$ satisfy the following conditions:

(i) $\alpha$ is a dual molecule;

(ii) $\vee_{a \in L}\left(a \wedge a^{\prime}\right) \leq \alpha<I$;

(iii) there exists $\beta \in L$ such that $\beta \wedge\left(\beta \rightarrow \beta^{\prime}\right) \leq \alpha$.

Then $\alpha$ is called a appropriate resolution level.

Remark 2.1 It follows from [11] that the appropriate resolution level set in $L_{n 2}=L_{n} \times L_{2}$ is $\left\{\left(a_{k}, b_{2}\right) \mid(n+1) / 2 \leq k<(2 n-2) / 3\right\}$.

Theorem 2.1 [11] In $L P(X)$, if $\alpha$ is a appropriate resolution level, then the Weak Completeness Theorem of $\alpha$-resolution principle holds.

Theorem 2.2 (Equivalence between $\alpha$ resolution in $L P(X)$ and $\alpha$-resolution in $L F(X)$ ) In $L P(X), q \in D_{\alpha}(g)$ iff $Q \in D_{\alpha}(G)$ in $L F(X)$, where $q, g$ are ground instances of $Q, G$ respectively.

Proof. (Necessity) It is easily proved according to Theorem 11.4.6 (Lift Theorem) in [2]

(Sufficiency) Suppose $Q \in D_{\alpha}(G)$ in $L F(X)$, then for any interpretation $I, v(G \wedge Q) \leq \alpha$. Since by substituting $Q$ and $G$ will have no common variables, we assume that $Q$ and $G$ have no common variables without lose of generality. Let $q, g$ be ground instances of $Q, G$ respectively, $u$ their corresponding 
interpretation. For any interpretation $I$ in which $u$ is part of interpretation of constants and functions in $Q, G, v(g \wedge q) \leq \alpha$. Therefore, for any $H$ interpretation $I_{H}, v_{H}(g \wedge q) \leq \alpha$.

Remark 2.2 It follows from Theorem 2.2 that the determination of $\alpha$-resolution in $\operatorname{LF}(X)$ can be equivalently transformed into that of $\alpha$-resolution in $L P(X)$. So the focus is given on the determination of $\alpha$-resolution in $L P(X)$.

In order to deal with widely used linguistic values in intelligent information processing with the base of logical framework, in [9,11] a linguistic truth-valued LIA $L_{n} \times L_{2}\left(=L_{n 2}\right)$ and the corresponding linguistic truth-valued propositional logic $L_{n 2} P(X)$ were investigated.

Theorem $2.3 v$ is a valuation in $L_{n m} P(X)$ iff there exist valuations $\mu$ and $\sigma$ in $L_{n} P(X)$ and $L_{m} P(X)$ respectively such that $v=\mu \times \sigma$, where $L_{n m}=L_{n} \times L_{m}$.

Proof. Notice that any symbol $p$ in $L P(X)$ can be seen as different logical formula when $p$ is interpretated in different valuation field $L$.

(Necessity). Let $v$ be a valuation in $L_{n m} P(X)$.

(1) for arbitrary a $p \in L_{n} P(X)$, it is a formula in $L_{n m} P(X)$ when it is interpreted in the valuation field $L_{n m}$. Let $\mu(p)=\left.v(p)\right|_{L_{n}}$, then it is easily proved that $\mu$ is a valuation in $L_{n} P(X)$.

(2) for arbitrary $p \in L_{m} P(X)$, it is a formula in $L_{n m} P(X)$ when it is interpreted in the valuation field $L_{n m}$. Let $\sigma(p)=\left.v(p)\right|_{L_{m}}$, then it is easily proved that $\sigma$ is a valuation in $L_{m} P(X)$. Hence, for arbitrary a $p \in L_{n m} P(X)$,

$v(p)=\left(\left.v(p)\right|_{L_{n}},\left.v(p)\right|_{L_{m}}\right)=(\mu(p), \sigma(p))=(\mu \times \sigma)(p)$.

(Sufficiency) It only needs to prove that for arbitrary formula $p, q$ in $L_{n m} P(X)$,

$$
\begin{aligned}
& v(p \rightarrow q)=v(p) \rightarrow v(q) . \text { In fact, } \\
& \begin{aligned}
v(p \rightarrow q) & =(\mu \times \sigma)(p \rightarrow q) \\
& =(\mu(p \rightarrow q), \sigma(p \rightarrow q)) \\
& =(\mu(p) \rightarrow \mu(q), \sigma(p) \rightarrow \sigma(q)) \\
& =(\mu(p), \sigma(p)) \rightarrow(\mu(q), \sigma(q)) \\
& =(\mu \times \sigma)(p) \rightarrow(\mu \times \sigma)(q)=v(p) \rightarrow v(q) .
\end{aligned}
\end{aligned}
$$

Theorem 2.4 In $L_{n} \times L_{2}=L_{n 2}$, let $\alpha=\left(a_{k}, b_{2}\right)$ $((n+1) / 2 \leq k<(2 n-2) / 3) \quad, \quad g \in L_{n 2} P(X)$. Then $t \in D_{\alpha}(g)=\left\{h \in L_{n 2} P(X): g \wedge h \leq \alpha\right\}$

iff $\left.\left.t\right|_{L_{n} P(X)} \in D_{\alpha}(g)\right|_{L_{n} P(X)}$

$$
=\left\{\left.h\right|_{L_{n} P(X)}:\left.\left.g\right|_{L_{n} P(X)} \wedge h\right|_{L_{n} P(X)} \leq a_{k}\right\},
$$

where $\left.p\right|_{L_{n} P(X)}$ ) denotes the restriction of $p$ on $L_{n} P(X)$.

Proof. In $L_{n} \times L_{2}=L_{n 2} \quad, \alpha=\left(a_{k}, b_{2}\right)$ $((n+1) / 2 \leq k<(2 n-2) / 3)$, $t \in D_{\alpha}(g)=\left\{h \in L_{n 2} P(X) \mid g \wedge h \leq \alpha\right\}$

iff for arbitrary a valuation $v$ in $L_{n 2} P(X)$, $v(g \wedge t) \leq \alpha$

iff for arbitrary a valuation $v$ in $L_{n 2} P(X)$, $v(g) \wedge v(t) \leq \alpha \quad$ (According to Theorem 2.3, if $v=\mu \times \sigma \quad, \quad$ then $\quad v(g)=(\mu(g), \sigma(g))$, $v(t)=(\mu(t), \sigma(t)))$

iff for arbitrary a valuation $\mu$ in $L_{n} P(X)$, $\mu(g) \wedge \mu(t) \leq a_{k}$

iff $\left.\left.t\right|_{L_{n} P(X)} \in D_{\alpha}(g)\right|_{L_{n} P(X)}=$

$$
\left\{\left.h\right|_{L_{n} P(X)}:\left.\left.g\right|_{L_{n} P(X)} \wedge h\right|_{L_{n} P(X)} \leq a_{k}\right\} \text {. }
$$

Remark 2.3 It follow from Theorem 2.4 that $\alpha=\left(a_{k}, b_{2}\right)$-resolution in the linguistic truth-valued propositional logic $L_{n 2} P(X)$ based on LIA is equivalent to $a_{k}$-resolution in the linguistic truthvalued propositional logic $L_{n} P(X)$ based on LIA, where $(n+1) / 2 \leq k<(2 n-2) / 3$.

From the above analysis, for $(n+1) / 2 \leq k<(2 n-2) / 3, \alpha=\left(a_{k}, b_{2}\right)$-resolution in the linguistic truth-valued first-order logic $L_{n 2} F(X)$ can be equivalently transformed into that in in the linguistic truth-valued propositional logic $L_{n 2} P(X)$, and further can be equivalently transformed to the $a_{k}$ resolution in the linguistic truth-valued propositional logic $L_{n} P(X)$.

\section{Typical inequality of generalized literals}

Theorem 3.1 Let $F\left(x_{1}, \cdots, x_{m}\right)$ be an s-IESF with propositional $x_{1}, \ldots, x_{m}$ and constants $a_{1}, \ldots, a_{t}$. Then there exist literals $p$ and $q$, and constant $a$ such that

(1) $F\left(x_{1}, \cdots, x_{m}\right) \geq p \rightarrow q$, or

(2) $F\left(x_{1}, \cdots, x_{m}\right) \geq a \rightarrow p$, or

(3) $F\left(x_{1}, \cdots, x_{m}\right) \geq(p \rightarrow q)^{\prime}$, or

(4) $F\left(x_{1}, \cdots, x_{m}\right) \geq(a \rightarrow p)^{\prime}$.

Proof. The conclusion is straightforward when $s=1$ or $\mathrm{s}=2$.

When $\mathrm{s}>2$, by Lemma 11.3.2 in [2], there exist ( $\mathrm{s}-2)$ 
IESF $G_{1}\left(x_{1}, \cdots, x_{m}\right)$ and 1-IESF $G_{2}\left(x_{1}, \cdots, x_{m}\right)$ such that

$$
\begin{aligned}
& F\left(x_{1}, \cdots, x_{m}\right)=G_{1}\left(x_{1}, \cdots, x_{m}\right) \rightarrow G_{2}\left(x_{1}, \cdots, x_{m}\right) \geq \\
& G_{2}\left(x_{1}, \cdots, x_{m}\right) .
\end{aligned}
$$

Notice that there exist literals $p$ and $q$, and constant $a$ such that

$$
\begin{aligned}
& G_{2}\left(x_{1}, \cdots, x_{m}\right)=p \rightarrow q, \text { or } \\
& G_{2}\left(x_{1}, \cdots, x_{m}\right)=a \rightarrow q, \text { or } \\
& G_{2}\left(x_{1}, \cdots, x_{m}\right)=(p \rightarrow q)^{\prime}, \text { or } \\
& G_{2}\left(x_{1}, \cdots, x_{m}\right)=(a \rightarrow p)^{\prime} .
\end{aligned}
$$

It follows the conclusion.

Theorem 3.2 Let $F\left(x_{1}, \cdots, x_{m}\right)$ be an s-IESF with propositional $x_{1}, \ldots, x_{m}$ and constants $a_{1}, \ldots, a_{t}$. Then

(1) there exists a valuation $v$ such that $v\left(F\left(x_{1}, \cdots, x_{m}\right)\right)=I$, or

(2) there exists a constant $a$, for arbitrary constant $b$, when $a \geq b$, there exists a valuation $v$ such that $v\left(F\left(x_{1}, \cdots, x_{m}\right)\right) \geq b$.

Proof. (1). By Theorem 3.1, there exist literals $p$ and $q$, and constant $a$ such that

(i) $F\left(x_{1}, \cdots, x_{m}\right) \geq p \rightarrow q$, or

(ii) $F\left(x_{1}, \cdots, x_{m}\right) \geq a \rightarrow p$, or

(iii) $F\left(x_{1}, \cdots, x_{m}\right) \geq(p \rightarrow q)^{\prime}$,

For (i), by the proof of Theorem 3.1, $p \neq q$, then there exists a valuation $v$ such that $v(q)=I$, so $v(F)=I$.

For (ii), there exists a valuation $v$ such that $v(p)=I$, so $v(F)=I$.

For (iii), by the proof of Theorem $3.1, p \neq q$, then there exists a valuation $v$ such that $v(p)=I, v(q)=O$, so $v(F)=I$.

(2) By Theorem 3.1, there exist literal $p$ and constant $a$ such that $F\left(x_{1}, \cdots, x_{m}\right) \geq(a \rightarrow p)^{\prime}$. For arbitrary a constant $b$, when $a \geq b$, there exists a valuation $v$ such that $v(p)=O$, it follows that $v\left(F\left(x_{1}, \cdots, x_{m}\right)\right) \geq b$.

Theorem 3.3 Let $F\left(x_{1}, \cdots, x_{m}\right)$ be an IESF with propositonals $X_{1}, \ldots, x_{m}$ and without any constant, then there exists a valuation $v$ such that $v\left(F\left(x_{1}, \cdots, x_{m}\right)\right)=I$.

Proof. The forms of $F\left(x_{1}, \cdots, x_{m}\right)$ are only (1) or (3) in Theorem 3.1. By theorem 3.2, it concludes the proof.

\section{The determination table of $\alpha$ - resolution in $L P(X)$}

In $L P(X)$, we denote the sets of all the constants, all the propositional variables and their negations, and all the generalized literals by $C, \bar{L}$ and $W$ respectively. Let

$W_{1}=\{f \mid f$ be a generalized literal, and there exist $r, s \in \bar{L}, r \neq s$ such that $f \geq r \rightarrow s\}$.

$W_{2}=\{f \mid f$ be a generalized literal, and there exist $a \in C, \quad s \in \bar{L}$ such that $f \geq a \rightarrow s\}$.

$W_{3}=\{f \mid f$ be a generalized literal, and there exist $r, s \in \bar{L}, r \neq s$ such that $\left.f \geq(r \rightarrow s)^{\prime}\right\}$.

$W_{4}=\{f \mid f$ be a generalized literal, and there exist $a \in C, s \in \bar{L}$ such that $\left.f \geq(a \rightarrow s)^{\prime}\right\}$.

By Theorem 3.1, $W_{1} \cup W_{2} \cup W_{3} \cup W_{4}=W$.

Theorem 4.1 Let $\alpha \in C, g \in L P(X)$ and $D_{\alpha}(g)=\{h \in L P(X) \mid h \wedge g \leq \alpha\}$ (Noted: $h \in D_{\alpha}(g)$ iff $\left.g \in D_{\alpha}(h)\right)$. We can get the following $\alpha$ resolution table:

\section{Case [1]:}

(i) Let $\underset{t \in L}{\vee}\left(t \wedge t^{\prime}\right) \leq \alpha$. If $h=\left(g^{\prime} \rightarrow g\right)^{\prime}$ or $\left(g^{\prime} \rightarrow q\right)^{\prime}$ or $(p \rightarrow g)^{\prime}$, then $h \in D_{\alpha}(g)$;

(ii) If $h>\left(g^{\prime} \rightarrow g\right)^{\prime}$ and $d \not \alpha \alpha$ where $d$ is the maximum value of $g \wedge\left(g^{\prime} \rightarrow g\right)^{\prime}$, or $h>\left(g^{\prime} \rightarrow q\right)^{\prime}$ and $d \not \leq \alpha$, where $d$ is the maximum value of $g \wedge\left(g^{\prime} \rightarrow q\right)^{\prime}$, then $h \notin D_{\alpha}(g)$;

(iii) Others, $h \notin D_{\alpha}(g)$.

\section{Case [2]:}

(i) Let $\underset{t \in L}{\vee}\left(t \wedge t^{\prime}\right) \leq \alpha$. If $h=(a \rightarrow g)^{\prime}$, then $h \in D_{\alpha}(g)$;

(ii) If $h>(a \rightarrow g)^{\prime}$ and $d \not \alpha \alpha$, where $d$ is the maximum value of $g \wedge(a \rightarrow g)^{\prime}$, then $h \notin D_{\alpha}(g)$;

(iii) Others, if $a \not \alpha$, then $h \notin D_{\alpha}(g)$.

\section{Case [3]:}

(i) Let $\underset{t \in L}{\vee}\left(t \wedge t^{\prime}\right) \leq \alpha$. If $g=r \rightarrow s, h=(r \rightarrow s)^{\prime}$, then $h \in D_{\alpha}(g)$;

(ii) If $g>r \rightarrow s$ or $h>(r \rightarrow s)^{\prime}$ and $d \not \alpha \alpha$, where $d$ is the maximum value of $(r \rightarrow s) \wedge(r \rightarrow s)^{\prime}$, then $h \notin D_{\alpha}(g)$;

(iii) If there exists $\beta \in L$ such that $\beta \wedge\left(\beta \rightarrow \beta^{\prime}\right) \not \leq \alpha$, and $g \geq r \rightarrow r^{\prime}$ or $h \geq(r \rightarrow q)^{\prime}$, then $h \notin D_{\alpha}(g)$.

(iv) Others, $h \notin D_{\alpha}(g)$.

\section{Case [4]:}


(i) If $g \geq r \rightarrow r^{\prime}, h \geq\left(a \rightarrow r^{\prime}\right)^{\prime}$, and $d \not \alpha \alpha$, where $d$ is the maximum value of $\left(r \rightarrow r^{\prime}\right) \wedge\left(a \rightarrow r^{\prime}\right)^{\prime}$, then $h \notin D_{\alpha}(g)$;

(ii) Others, if $a \npreceq \alpha$, then $h \notin D_{\alpha}(g)$. (i) If $g \geq b \rightarrow s, h \geq a \rightarrow s^{\prime}$, and $d \leq \alpha$, where $d$ is the maximum value of $(b \rightarrow s) \wedge\left(a \rightarrow s^{\prime}\right)$, then $h \notin D_{\alpha}(g)$;

(ii) Others, $h \notin D_{\alpha}(g)$.

\section{Case [5]:}

\begin{tabular}{|c|c|c|c|c|c|c|c|c|}
\hline \multicolumn{2}{|c|}{$g \wedge \lambda h$} & \multicolumn{2}{|l|}{$\in C$} & \multirow[t]{2}{*}{$\in L$} & \multirow[t]{2}{*}{$\geq p \rightarrow q$} & \multirow[t]{2}{*}{$\geq a \rightarrow q$} & \multirow[t]{2}{*}{$\geq(p \rightarrow q)^{\prime}$} & \multirow[t]{2}{*}{$\geq(a \rightarrow q)^{\prime}$} \\
\hline & & $\leq \alpha$ & $\not \leq \alpha$ & & & & & \\
\hline \multirow[t]{2}{*}{$\in C$} & $\leq \alpha$ & Yes & Yes & Yes & Yes & Yes & Yes & Yes \\
\hline & $\not \alpha \alpha$ & Yes & $\begin{array}{l}\text { If } \alpha \text { is a } \\
\text { dual } \\
\text { molecule } \\
\text {, then No }\end{array}$ & No & No & No & No & $\begin{array}{l}\text { If } \alpha \text { is a } \\
\text { dual } \\
\text { molecule, } \\
\text { and } \\
a \nless \alpha \alpha \\
\text { then No. }\end{array}$ \\
\hline \multicolumn{2}{|l|}{$\in L$} & Yes & No & $\begin{array}{l}\text { If } \\
\vee_{t \in L}\left(t \wedge t^{\prime}\right) \leq \alpha \\
, h=g^{\prime} \\
\text { then Yes; } \\
\text { others, No. }\end{array}$ & $\begin{array}{l}\text { If } \\
h=g \rightarrow g^{\prime} \\
\text { and there } \\
\text { exists } \beta \in L, \\
\beta \wedge\left(\beta \rightarrow \beta^{\prime}\right) \\
\leq \alpha, \text { then } \\
\text { No; others, } \\
\text { No }\end{array}$ & $\begin{array}{l}\text { If } q=g^{\prime} \\
\text { and } a^{\prime} \nless \alpha \alpha, \\
\text { then No; } \\
\text { others, No. }\end{array}$ & {$[1]$} & {$[2]$} \\
\hline \multicolumn{2}{|c|}{$\geq r \rightarrow s$} & Yes & No & $\begin{array}{l}\text { If } \\
g=h \rightarrow h^{\prime} \\
\text { and there } \\
\text { exists } \beta \in L \\
\beta \wedge\left(\beta \rightarrow \beta^{\prime}\right) \\
\leq \alpha, \text { then } \\
\text { No; others, } \\
\text { No. }\end{array}$ & $\begin{array}{l}\text { If } \\
g \geq r \rightarrow r^{\prime}, \\
h \geq r^{\prime} \rightarrow r, \\
\text { and there } \\
\text { exists, } \beta \in L \\
\beta \wedge\left(\beta \rightarrow \beta^{\prime}\right) \\
\leq \alpha, \text { then } \\
\text { No; others, } \\
\text { No }\end{array}$ & $\begin{array}{l}\text { If } \\
g \geq r \rightarrow r^{\prime}, \\
h \geq a \rightarrow r, \\
\text { and there } \\
\text { exists } \beta \in L, \\
\beta \wedge\left(\beta \rightarrow \beta^{\prime}\right) \\
\not \alpha, \text { then } \\
\text { No; others, } \\
\text { No }\end{array}$ & [3] & [4] \\
\hline \multicolumn{2}{|c|}{$\geq b \rightarrow s$} & Yes & No & $\begin{array}{l}\text { If } s=h^{\prime}, \\
\text { and } b^{\prime} \leq \alpha, \\
\text { then No; } \\
\text { others, No. }\end{array}$ & $\begin{array}{l}\text { If } \\
h \geq p \rightarrow p^{\prime}, \\
g \geq b \rightarrow p, \\
\text { and there } \\
\text { exists } \beta \in L, \\
\beta \wedge\left(\beta \rightarrow \beta^{\prime}\right) \\
\leq \alpha, \text { then } \\
\text { No; others, } \\
\text { No. }\end{array}$ & {$[5]$} & [6] & [7] \\
\hline \multicolumn{2}{|c|}{$\geq(r \rightarrow s)^{\prime}$} & Yes & No & $\begin{array}{l}{[8] \text { is similar }} \\
\text { to }[1]\end{array}$ & $\begin{array}{l}\text { [9] is similar } \\
\text { to [3] }\end{array}$ & $\begin{array}{l}{[10] \text { is }} \\
\text { similar to [6] }\end{array}$ & [11] & [12] \\
\hline \multicolumn{2}{|c|}{$\geq(b \rightarrow s)^{\prime}$} & Yes & $\begin{array}{l}\text { If } \alpha \text { is a } \\
\text { dual } \\
\text { molecule } \\
\text {, and } \\
b \leq \alpha, \\
\text { then } \\
\text { No }\end{array}$ & $\begin{array}{l}{[13] \text { is }} \\
\text { similar to [2] }\end{array}$ & $\begin{array}{l}\text { [14 is similar } \\
\text { to }[4]\end{array}$ & $\begin{array}{l}{[15] \text { is }} \\
\text { similar to [7] }\end{array}$ & $\begin{array}{l}{[16] \text { is }} \\
\text { similar to } \\
{[12]}\end{array}$ & [17] \\
\hline
\end{tabular}


Table 1: The determination table of $\alpha$-resolution in $L P(X)$.

Remark 4.1: In Table 1, "Yes" represents that $g$ and $h$ are resolvent while "No" represents that $g$ and $h$ are not resolvent.

Case [6]:

(i) If $g \geq b \rightarrow s, h \geq\left(s^{\prime} \rightarrow q\right)^{\prime}$, and $d \not \alpha \alpha$, where $d$ is the maximum value of $(b \rightarrow s) \wedge\left(s^{\prime} \rightarrow q\right)^{\prime}$, then $h \notin D_{\alpha}(g)$;

(ii) Others, $h \notin D_{\alpha}(g)$.

\section{Case [7]:}

(i) If $g \geq b \rightarrow s, h \geq(a \rightarrow s)^{\prime}$, and $d \leq \alpha$, where $d$ is the maximum value of $(b \rightarrow s) \wedge(a \rightarrow s)^{\prime}$, then $h \notin D_{\alpha}(g)$;

(ii) Others, if $a \nless \alpha$, then $h \notin D_{\alpha}(g)$.

\section{Case[8]:}

(i) Let $\bigvee_{t \in L}\left(t \wedge t^{\prime}\right) \leq \alpha$. If $g=\left(h^{\prime} \rightarrow h\right)^{\prime}$ or $\left(h^{\prime} \rightarrow s\right)^{\prime}$ or $(r \rightarrow h)^{\prime}$, then $h \in D_{\alpha}(g)$;

(ii) If $g>\left(h^{\prime} \rightarrow h\right)^{\prime}$ and $d \not \alpha$, where $d$ is the maximum value of $h \wedge\left(h^{\prime} \rightarrow h\right)^{\prime}$, or $g>\left(h^{\prime} \rightarrow s\right)^{\prime}$ and $d \not \alpha$ where $d$ is the maximum value of $h \wedge\left(h^{\prime} \rightarrow s\right)^{\prime}$, then $h \notin D_{\alpha}(g)$;

(iii) Others, $h \notin D_{\alpha}(g)$.

\section{Case[9]:}

(i) Let $\bigvee_{t \in L}\left(t \wedge t^{\prime}\right) \leq \alpha$. If $h=p \rightarrow q, g=(p \rightarrow q)^{\prime}$, then $h \in D_{\alpha}(g)$;

(ii) If $h>p \rightarrow q$ or $g>(p \rightarrow q)^{\prime}$ and $d \not \alpha \alpha$, where $d$ is the maximum value of $(p \rightarrow q) \wedge(p \rightarrow q)^{\prime}$, then $h \notin D_{\alpha}(g)$;

(iii) If there exists $\beta \in L$ such that $\beta \wedge\left(\beta \rightarrow \beta^{\prime}\right) \not \leq \alpha$, and $h \geq p \rightarrow p^{\prime}$ or $g \geq(p \rightarrow s)^{\prime}$, then $h \notin D_{\alpha}(g)$.

(iv) Others, $h \notin D_{\alpha}(g)$.

\section{Case [10]:}

(i) If $h \geq a \rightarrow q, g \geq\left(q^{\prime} \rightarrow s\right)^{\prime}$, and $d \not \leq \alpha$ where $d$ is the maximum value of $(a \rightarrow q) \wedge\left(q^{\prime} \rightarrow s\right)^{\prime}$, then $h \notin D_{\alpha}(g)$;

(ii) Others, $h \notin D_{\alpha}(g)$.

\section{Case [11]:}

(i) If $g \geq\left(r \rightarrow r^{\prime}\right)^{\prime}, h \geq\left(r^{\prime} \rightarrow q\right)^{\prime}$ and $d \not \alpha \alpha$, where $d$ is the maximum value of $\left(r \rightarrow r^{\prime}\right)^{\prime} \wedge\left(r^{\prime} \rightarrow q\right)^{\prime}$, then $h \notin D_{\alpha}(g)$;

(ii) If $g \geq(r \rightarrow s)^{\prime}, h \geq\left(r \rightarrow s^{\prime}\right)^{\prime}$ and $d \not \alpha \alpha$, where $d$ is the maximum value of $(r \rightarrow s)^{\prime} \wedge\left(r \rightarrow s^{\prime}\right)^{\prime}$, then $h \notin D_{\alpha}(g)$;

(iii) If $g=(r \rightarrow s)^{\prime}, h=\left(r^{\prime} \rightarrow s^{\prime}\right)^{\prime}$, then $g \wedge h=O$. (iv) If $g>(r \rightarrow s)^{\prime}$, or $h>\left(r^{\prime} \rightarrow s^{\prime}\right)^{\prime}$, then we can not judge if $h$ belongs to $D_{\alpha}(g)$;

(v) If $g \geq(r \rightarrow s)^{\prime}, h \geq\left(r^{\prime} \rightarrow q\right)^{\prime}$ and $d \not \leq \alpha$, where $d$ is the maximum value of $(r \rightarrow s)^{\prime} \wedge\left(r^{\prime} \rightarrow q\right)^{\prime}$, then $h \notin D_{\alpha}(g)$;

(vi) If $g \wedge h \geq(r \rightarrow s)^{\prime}, \quad g \wedge h \geq(s \rightarrow q)^{\prime}, \quad$ and $d \not \alpha$ where $d$ is the maximum value of $(r \rightarrow s)^{\prime} \wedge(s \rightarrow q)^{\prime}$, then $h \notin D_{\alpha}(g)$;

(vii) Others, $h \notin D_{\alpha}(g)$.

\section{Case[12]:}

(i) If $g \geq\left(r \rightarrow r^{\prime}\right)^{\prime}, h \geq(a \rightarrow r)^{\prime}$ and $d \not \leq \alpha$, where $d$ is the maximum value of $\left(r \rightarrow r^{\prime}\right)^{\prime} \wedge(a \rightarrow r)^{\prime}$, then $h \notin D_{\alpha}(g)$;

(ii) If $g \geq\left(r \rightarrow r^{\prime}\right)^{\prime}, \quad h \geq(a \rightarrow q)^{\prime}$ and $a \not \alpha$, then $h \notin D_{\alpha}(g)$;

(iii) If $g \geq(r \rightarrow s)^{\prime}, h \geq(a \rightarrow r)^{\prime}$ and $d \not \leq \alpha$, where $d$ is the maximum value of $(r \rightarrow s)^{\prime} \wedge(a \rightarrow r)^{\prime}$, then $h \notin D_{\alpha}(g)$;

(iv) If $g \geq(r \rightarrow s)^{\prime}, \quad h \geq(a \rightarrow q)^{\prime}$ and $a \not \alpha$, then $h \notin D_{\alpha}(g)$.

\section{Case[13]:}

(i) Let $\underset{t \in L}{\vee}\left(t \wedge t^{\prime}\right) \leq \alpha$. If $g=(b \rightarrow h)^{\prime}$, then $h \in D_{\alpha}(g)$;

(ii) If $g>(b \rightarrow h)^{\prime}$ and $d \not \alpha$, where $d$ is the maximum value of $h \wedge(b \rightarrow h)^{\prime}$, then $h \notin D_{\alpha}(g)$;

(iii) Others, if $b \nless \alpha$, then $h \notin D_{\alpha}(g)$.

\section{Case [14]:}

(i) If $h \geq p \rightarrow p^{\prime}, g \geq\left(b \rightarrow p^{\prime}\right)^{\prime}$, and $d \not \leq \alpha$ where $d$ is the maximum value of $\left(p \rightarrow p^{\prime}\right) \wedge\left(b \rightarrow p^{\prime}\right)^{\prime}$, then $h \notin D_{\alpha}(g)$;

(ii) Others, if $b \leq \alpha$, then $h \notin D_{\alpha}(g)$.

\section{Case [15]:}

(i) If $h \geq a \rightarrow q, g \geq(b \rightarrow q)^{\prime}$ and $d \not \alpha \alpha$, where $d$ is the maximum value of $(a \rightarrow q) \wedge(b \rightarrow q)^{\prime}$, then $h \notin D_{\alpha}(g)$;

(ii) Others, if $b \npreceq \alpha$, then $h \notin D_{\alpha}(g)$.

\section{Case [16]:}

(i) If $h \geq\left(p \rightarrow p^{\prime}\right)^{\prime}, g \geq(b \rightarrow p)^{\prime}$ and $d \not \alpha \alpha$, where $d$ is the maximum value of $\left(p \rightarrow p^{\prime}\right)^{\prime} \wedge(b \rightarrow p)^{\prime}$, then $h \notin D_{\alpha}(g)$;

(ii) If $h \geq\left(p \rightarrow p^{\prime}\right)^{\prime}, \quad g \geq(b \rightarrow s)^{\prime}$ and $b \leq \alpha$, then $h \notin D_{\alpha}(g)$; 
(iii) If $h \geq(p \rightarrow q)^{\prime}, g \geq(b \rightarrow p)^{\prime}$ and $d \not \alpha \alpha$, where $d$ is the maximum value of $(p \rightarrow q)^{\prime} \wedge(b \rightarrow p)^{\prime}$, then $h \notin D_{\alpha}(g)$;

(iv) If $h \geq(p \rightarrow q)^{\prime}, \quad g \geq(b \rightarrow s)^{\prime}$ and $b \leq \alpha$, then $h \notin D_{\alpha}(g)$.

Case[17]:

(i) If $g \geq(b \rightarrow s)^{\prime}, h \geq(a \rightarrow s)^{\prime}$ and $b \wedge a \leq \alpha$, 则 $h \notin D_{\alpha}(g)$;

(ii) If $g \geq(b \rightarrow s)^{\prime}, h \geq\left(a \rightarrow s^{\prime}\right)^{\prime}$ and $d \not \alpha \alpha$, where $d$ is the maximum value of $(b \rightarrow s)^{\prime} \wedge\left(a \rightarrow s^{\prime}\right)^{\prime}$, then $h \notin D_{\alpha}(g)$.

Remark 4.2 It follows from Theorem 4.1 that whether two generalized literals $g, h$ are resolvent can be checked through the determination table of $\alpha$ resolution in $L P(X)$.

\section{Conclusions}

In this paper, the following results were obtained:

(1) The difficult and key problem of $\alpha$-resolution of any two literals or two generalized literals in latticevalued first-order logic $L F(X)$ based on lattice implication algebra can be transformed equivalently to that for lattice-valued propositional $\operatorname{logic} L P(X)$, which much simplified the problem.

(2) $\alpha$-resolution in linguistic truth-valued propostional logic $L_{n 2} P(X)$ based on lattice implication algebra can be transformed equivalently into $\alpha$-resolution for linguistic truth-valued propostional logic $L_{n} P(X)$ based on lattice implication algebra. Hence $\alpha$-resolution of two literals or two generalized literals in linguistic truth-valued first-order logic $L_{n 2} F(X)$ can be transformed into $\alpha$-resolution of two literals or two generalzied literals in linguistic truth-valued propositional logic $L_{n} P(X)$.

(3) The provided determination table with 49 cases for two generalized literals in lattice-valued propositional logic $L P(X)$ based on lattice implication algebra provided criteria to determine if any two literals or two generalized literals are $\alpha$-resolution pair in $L P(X)$.

\section{Acknowledgement}

This work is partially supported by the National Natural Science Foundation of P.R. China (Grant No. 60474022), the Specialized Research Foundation for the Doctoral Program of Higher Education of China (Grant No. 20060613007), and the China-Flanders bilateral scientific cooperation joint project (Grant No. 011S1105).

\section{References}

[1] Y. Xu, Lattice implication algebra, Journal of Southwest Jiaotong University. 1:20-27, 1993.

[2] Y. Xu, D. Ruan, K.Y. Qin and J. Liu, Latticevalued logic, Springer-verlag. Berlin., 2003.

[3] Y. $\mathrm{Xu}$ and K.Y. Qin, Lattice-valued propositional logic( I ), Journal of Southwest Jiaotong University, 1:123-128, 1993.

[4] K.Y. Qin and Y. Xu, Lattice-valued propositional logic( II) , Journal of SouthwestJiaotong University, 2:22-27, 1994.

[5] Y. Xu, D. Ruan, E.E. Kerre and J. Liu, $\alpha$-resolution principle based on lattice-valued logic $L P(X)$, Information Science, 130:195223, 2000.

[6] Y. Xu, D. Ruan, E.E. Kerre and J. Liu, $\alpha$ - automated reasoning method based on $L P(X)$, Proceedings of East West Fuzzy Colloquium 2000 and 8th, Germany, 2000.

[7] Y. Xu, D. Ruan, E.E. Kerre and J. Liu, $\alpha$ - resolution principle based on first-order lattice-valued logic $L F(X)$, Information Science, 132:221-239, 2001.

[8] J. Liu, Study on lattice-valued logic system and lattice-valued resolution based on lattice implication algebras, Ph.D., Thesis, Southwest Jiaotong University, 1999.(in Chinese)

[9] Y. Xu, S.W. Chen and J. Ma, Linguistic truthvalued lattice implication algebra and its properties, IMACS Multiconference on Computational Engineering in System Application, pp.1413-1418, 2006.

[10] Y. Xu, J. Liu, D. Ruan and T.T. Lee, On the consistency of rule-bases based on lattice valued first-order logic $L F(X)$, International Journal of Intelligent System, 21:399-424, 2006.

[11] Y. Xu, S. W. Chen, J. Liu and D. Ruan, Weak completeness of resolution in a lattice truthvalued propositional logic, IFSA 2007.

[12] Y. Xu, K.Y. Qin and Z.M. Song, On syntax of first-order lattice-valued logic system FM, Chinese Science Bulletin, 42 (17):1052-1055, 1997. ( English version).

[13] Y. Xu, K.Y. Qin, J. Liu and Z.M. Song, L valued propositional logic $L_{v p l}$, Information Sciences, 114:205-235, 1999.

[14] Y. Xu, Z.M. Song, K.Y. Qin and J. Liu, On syntax of $L$-valued first-order logic $L_{v f l}$, International Journal of Multiple-valued logic, 7(7):213-257, 2001.

[15] Y. Xu, D. Ruan, E.E. Kerre and J. Liu, Approximate reasoning based on lattice-valued propositional logic $L_{v p l}$, in: D. Ruan, E.E. Kerre eds, Fuzzy IF-THEN Rule in 
Computational Intelligence: Theory and Application, Kluwer Academic Publishers, Dordrecht, pp.81-106, 2000.

[16] Schmitt and P.H., Computational aspects of three-valued logic, In: J.H. Siek-mann(Ed.) Proc. of the Eighth International Conference on Automated Deduction. Springer, Berlin, pp. 190-198, 1986.

[17] D. Meng, Y. Xu and H. D. Jia, Resolution principle based on six lattice-valued first-order logic $\mathrm{L}_{6} \mathrm{~F}(\mathrm{X})$, Proceedings of 2005 IEEE Networking, Sensing and Control Conference, pp. 838-843, 2005.

[18] D. Meng, X.F. Wang, Y. Xu and K.Y. Qin, Resolution Principle Based on Six Latticevalued Proposition Logic $L_{6} P(X)$, Proceedings of Second Intemational Conference on Machine and Cybernetics, pp. 2489-2494, 2003.

[19] J.P. Robison, A machine-oriented logic based on the resolution principle, J.ACM, 12, pp.2341, 1965

[20] D. Lehmann, On different concept of resolution, Zeit .f. Math. Logik and Grundlagen d. Math., 35, pp.71-77,1989.

[21] Z. Manna and R. Waldinger, Special relations in automated deduction, J.ACM, 33(1):1-59, 1986.

[22] H. Ganzinger and V.S. Stokkermans, Chaining techniques for automated theorem proving in man-valued logics, In: Proc. Of the 2000 ISMVL, Oregon, May, 23-25, IEEE Computer Society, Los Amamitos, pp.337-344, 2000.

[23] J. Liu, D. Ruan, Y. Xu and Z.M. Song, A resolution-like strategy based on a latticevalued logic, IEEE Transaction on Fuzzy System, 11(4):560-567, 2003.

[24] J. Ma, W.J. Li, D. Ruan and Y. Xu, FilterBased Resolution Principle for Lattice-Valued Propositional Logic LP(X), Information Sciences., 177, pp.1046-1062, 2007.

[25] J. Ma, S.W. Chen and Y. Xu, Fuzzy Logic from the Viewpoint of Machine Intelligence, Fuzzy Sets and Systems., 157:628-634, 2006.

[26] X.B. Li, D. Meng and Y. Xu. J-Automated Reasoning Method Based on LP(X). 2006 International Conference on Hybrid Information Technology, Nov. 9-11, Cheju Island, Korea, pp.141-144, 2006.

[27] L. Zou, J. Ma and Y. Xu, A framework of linguistic truth-valued propositional logic based on lattice implication algebra, Proceedings of 2006 IEEE International Conference on Granular Computing. May 10-12, Atlanta, USA, pp.574-577, 2006.
[28] L. Zou, X. Liu and Y. Xu, Resolution method of linguistic truth-valued propositional logic, Proceedings of 2005 International Conference on Neural Networks and Brain. 3:1996-1999, 2005.

[29] L. Zou, Pradip K. Das, Y. Xu and D. Meng, Soft-resolution method of linguistic hedges lattice-valued first-order logic, 8th Joint Conference on Information Sciences, Salt Lake City, Utah, USA. July21-26, 136-139, 2005.

[30] J.R. Stagle, Automated theorem proving with renamale and semantic resolution, J.ACM, 4(14), pp.687-697, 1967.

[31] G.A. Robinson and L. Wos, Paramodulation and theorem proving in first order theories with equality, Machine Ingelligence, 4, pp.135-150, 1969.

[32] D.W. Loveland, A liner format for resolution, Prof. IRIA Symp.Automatic Demonstration, pp.147-162, 1970 .

[33] C.L.Chang, The unit proof and the input proof in theorem proving, J.ACM, 17(4), pp.698-707, 1970.

[34] D. Smutna-Hlinena and P. Vojtas, Graded many-valued resolution with aggregation, Fuzzy Sets and System, 143:157-168, 2004. 